\title{
Discussão no rastreio de anormalidades renais em portador de apêndice pré-auricular com risco de alteração na organogênese: relato de caso
}

\section{Discussion on screening for renal abnormalities in patients with pre-auricular appendage at risk for changes in} organogenesis: case report

Discusión sobre el cribado de anomalías renales en un paciente con apéndice preauricular en riesgo de alteración de la organogénesis: reporte de caso

Bruno Antunes CONTRUCCI Pediatria, Hospital da Criança e Maternidade/ Faculdade de Medicina de São José do Rio Preto (FAMERP) 15090-000 São José do Rio Preto - SP, Brasil https://orcid.org/0000-0001-5507-4443

Gustavo Rogério PINATO

Pediatria, Hospital da Criança e Maternidade/Faculdade de Medicina de São José do Rio Preto (FAMERP) 15090-000 São José do Rio Preto - SP, Brasil https://orcid.org/0000-0003-1779-9376

Marina Vanzela Lania TELES

Neonatologia, Hospital da Criança e Maternidade/ Faculdade de Medicina de São José do Rio Preto (FAMERP) 15090-000 São José do Rio Preto - SP, Brasil https://orcid.org/0000-0002-0325-0190

Gabriella Maset da Silva FARIA Neonatologia, Hospital da Criança e Maternidade/ Faculdade de Medicina de São José do Rio Preto (FAMERP) 15090-000 São José do Rio Preto - SP, Brasil https://orcid.org/0000-0003-1689-4291

\section{Resumo}

A incidência de anormalidades congênitas do trato urinário associadas a alterações congênitas esporádicas em orelha externa é relatada na literatura, principalmente quando fatores de riscos pré-natais que modificam a embriogênese e organogênese fetal estão presentes. A presença de malformações renais predispõe a aumento da morbimortalidade relacionados a cálculos, infecções urinárias de repetição, sepse e doença renal crônica. Contudo, o rastreio de alterações renais, frente à presença de apêndice pré-auricular, não é consenso na literatura, mesmo quando há fatores que aumentam o risco para seu surgimento. Nesse contexto, a utilização da ultrassonografia abdominal em neonatos com apêndice pré-auricular e que possuem risco aumentado para anormalidades congênitas, assume destaque no diagnóstico precoce de condições que possam a vir, futuramente, causar repercussões clínicas. Assim, os autores buscaram relatar o caso de recém-nascido com malformação renal evidenciado em exame de imagem, o qual foi solicitado devido a apêndice pré-auricular esporádico evidenciado em exame físico associado à diabetes mellitus, hipertensão arterial sistêmica e tabagismo materno.

Descritores: Anormalidades Urogenitais; Rim Fundido; Organogênese; Pavilhão Auricular.

\section{Abstract}

The incidence of congenital urinary tract abnormalities associated with sporadic congenital changes in the external ear is reported in the literature, especially when prenatal risk factors that modify embryogenesis and fetal organogenesis are present. The presence of renal malformations predisposes to increased morbidity and mortality related to calculus, recurrent urinary tract infections, sepsis and chronic kidney disease. However, screening for renal changes, in the presence of a pre-auricular appendage, is not a consensus in the literature, even when there are factors that increase the risk for its appearance. In this context, the use of abdominal ultrasound in neonates with pre-auricular appendage and who are at increased risk for congenital abnormalities, is highlighted in the early diagnosis of conditions that may, in the future, cause clinical repercussions. Thus, the authors sought to report the case of a newborn with renal malformation evidenced in an image exam, which was requested due to sporadic pre-auricular appendage evidenced in physical examination associated with diabetes mellitus, systemic arterial hypertension and maternal smoking.

Descriptors: Urogenital Abnormalities; Fused Kidney; Organogenesis; Ear Auricle.

\section{Resumen}

La incidencia de anomalías congénitas del tracto urinario asociadas con cambios congénitos esporádicos en el oído externo se informa en la literatura, especialmente cuando están presentes factores de riesgo prenatales que modifican la embriogénesis y la organogénesis fetal. La presencia de malformaciones renales predispone a una mayor morbilidad y mortalidad relacionada con cálculos, infecciones recurrentes del tracto urinario, sepsis y enfermedad renal crónica. Sin embargo, el cribado de alteraciones renales, en presencia de un apéndice pre auricular, no es un consenso en la literatura, incluso cuando existen factores que aumentan el riesgo de su aparición. En este contexto, se destaca el uso de la ecografía abdominal en neonatos con apéndice pre auricular y con mayor riesgo de anomalías congénitas, en el diagnóstico precoz de patologías que, en el futuro, puedan tener repercusiones clínicas. Así, los autores buscaron reportar el caso de un recién nacido con malformación renal evidenciada en examen de imagen, el cual fue solicitado por apéndice pre auricular esporádico evidenciado en examen físico asociado a diabetes mellitus, hipertensión arterial sistémica y tabaquismo materno.

Descriptores: Anomalías Urogenitales; Riñón Fusionado; Organogénesis; Pabellón Auricular.

INTRODUÇÃOO

$$
\text { Os apêndices pré-auriculares são }
$$
anormalidades congênitas de orelha externa, comumente assintomática. A presença de tal apêndice, de forma esporádica e não relacionada a síndromes genéticas, apresenta associação com outras anomalias congênitas em cerca de um terço dos casos, entre eles as renais. De acordo com a literatura, acredita-se que possuem genes em comum, não havendo dados adicionais para corroborar tal fato ${ }^{1}$.
Aproximadamente $10 \%$ das crianças
nascem com malformações renais potencialmente significativas, onde a maior parte está relacionada com alterações estruturais assintomáticas, principalmente de trato urinário superior, sendo o rim em forma de ferradura o mais comum. Contudo, essa condição clínica relaciona-se, futuramente, com aumento da incidência de desenvolvimento de hidronefrose, infecções, formações calculosas ${ }^{2}$. 
$\mathrm{Na}$ faixa etária pediátrica, em especial os neonatos, as malformações renais se expressam, na maior parte das vezes, quando alterações irreversíveis decorrentes das alterações anatômicas já se fazem presente, como, por exemplo, a doença renal crônica decorrente de refluxo vesicoureteral, frequentemente relacionado a rim em ferradura. Além disso, sabe-se que alterações morfológicas de trato urinário geram predisposição à sepse, e em condições extremas, a morte ${ }^{3}$.

Em uma coorte prospectiva, evidenciouse que as correlações de malformações em orelha externa com alterações renais, se fizeram presentes em grande parte dos pacientes. Os autores concluíram que a incidência aumentava, principalmente quando vinculados a fatores de risco para alteração na organogênese fetal, sendo a história materna de diabetes mellitus gestacional e hipertensão arterial, incluídas como variáveis de associação importantes. Tal fato levou aos autores a sugerirem que a realização de ultrassonografia de abdome, em portadores de apêndices auriculares, fosse ponderada, principalmente quando relacionados a fatores associativos ${ }^{4}$.

Contudo, tal recomendação, de acordo com a literatura existente, é controversa e poucos estudos existem sobre o tema. Em um estudo retrospectivo envolvendo cerca de 20 mil crianças, o qual não foi ponderado a presença de fatores de risco relacionados a alteração na organogênese, a incidência de malformações renais em portadores de apêndice auricular isolado, apresentou-se de forma similar nos não portadores ${ }^{5}$.

A presença de hiperglicemia materna, níveis elevados de pressão arterial e tabagismo durante a gestação, são fatores sabiamente reconhecidos como influenciadores na embriogênese e organogênese fetal, predispondo a malformações congênitas ${ }^{6}$.

Assim, nota-se a importância de se debater e relatar casos sobre o tema, pouco encontrado na literatura, a fim de se chegar a um consenso sobre a investigação ultrassonográfica de malformação renal nos recém-nascidos, frente ao achado frequente e isolado no exame físico de apêndice auricular, associados ou não a fatores de risco para alteração no desenvolvimento fetal ${ }^{7}$.

CASO CLÍNICO

Genitora primigesta, 24 anos, hipertensa crônica, diabética tipo II previamente diagnosticada, com sinais de resistência periférica à insulina importante, com acantose nigricans e hirsutismo evidentes, apresentou-se com índice de massa corporal maior que 40 $\mathrm{kg} / \mathrm{m}^{2}$ durante os três trimestres da gestação. Possuía exame de hemoglobina glicada com valor de $11,5 \%$ no primeiro trimestre, além de tabagismo ativo pré-concepção. Fez uso de ácido acetilsalicílico, carbonato de cálcio, metildopa, metformina e insulina regular desde o início da gestação, com controle irregular de pressão arterial e glicemia. Ultrassonografias morfológicas e ecocardiograma fetal dentro dos parâmetros de normalidade, realizados conforme protocolo institucional. Não havia história familiar de doenças congênitas, síndromes familiares ou consanguinidade. Nascido de parto vaginal a termo, neonato do sexo masculino foi reanimado em sala de parto com ventilação por pressão positiva devido à hipotonia e ausência de drive respiratório, com melhora dos parâmetros clínicos, apresentando escala de APGAR 3/8. Ao exame físico primário, apresentou-se adequado para idade gestacional de acordo com o projeto INTERGROWTH-21 ${ }^{\text {ST }}$ e foi evidenciado apêndice pré-auricular em lado direito (Figura 1). Á palpação de abdome, não foi possível avaliar massas ou qualquer outra alteração, apresentando cordão umbilical com duas artérias e uma veia. Não havia qualquer outra anormalidade da propedêutica inicial ${ }^{7}$.

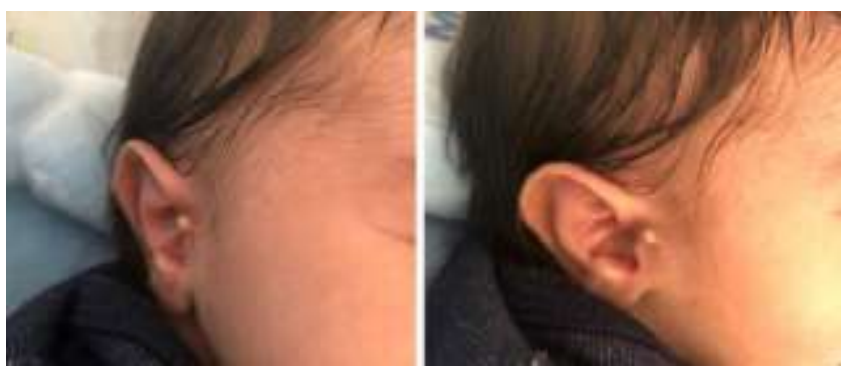

Figura 1: Apêndice pré-auricular, anteriormente à orelha externa direita.

Mantido em observação em temperatura adequada e sem desconforto respiratório, encaminhado ao alojamento conjunto materno estável clinicamente. Apresentou-se sem intercorrência durante primeiros dias de vida, sem alterações em padrões vitais, distensão abdominal ou massas palpáveis, diurese e evacuações presentes e diárias. Foram solicitados exames de rotina para filhos de mãe diabética, além de controle glicêmico nas primeiras horas de vida conforme protocolo institucional, com resultados dentro dos parâmetros de normalidade.

Foi solicitada ultrassonografia (US) abdominal no $2^{\circ}$ dia de vida devido à presença do apêndice pré-auricular associado a fatores de risco materno para malformações congênitas, apesar das semiologias urinária e abdominal estarem normais. Foi constatada a presença de fusão renal em polo inferior, com 
ureteres separados, sendo caracterizado como rim em ferradura, sem alteração de medula e córtex renal (Figura 2). Somado a tal achado, evidenciou-se uma imagem hipoecoica, ovalar, em topografia de adrenal direita, medindo 1,4 $\mathrm{cm} \times 0,9 \mathrm{~cm}$, com ausência de fluxo vascular ao estudo Doppler, sugerindo hemorragia adrenal.
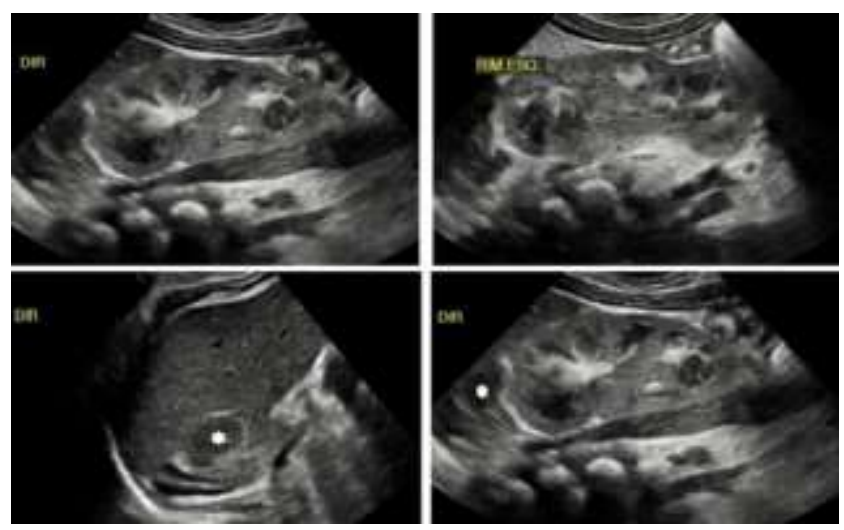

Figura 2: US no plano longitudinal, dos rins direito e esquerdo (imagens superiores). Fusão dos polos inferiores na linha mediana, anterior à veia cava inferior e aorta abdominal, configurando aspecto "em ferradura". Massas hipoecóicas adjacentes ao polo superior do rim direito, sugerindo hemorragia adrenal (asterisco branco, nas imagens inferiores).

Mediante a boa evolução clínica do paciente, recebeu alta hospitalar no $4^{\circ}$ dia de vida, com retorno agendado no ambulatório de Cirurgia Pediátrica para avaliação de anormalidade renal, e ambulatório de Pediatria Geral para realização de Teste de Triagem Auditiva Universal (Teste da Orelhinha), através de Emissões Otoacústicas Evocadas, sendo este último com resultado normal. No ambulatório de especialidades, neonato apresentou-se com um mês devido sem intercorrências clínicas, em bom estado geral, sem massas abdominais palpáveis em exame físico. Solicitado novo US de abdome para avaliação de evolução do achado ultrassonográfico prévio de imagem hipoecoica, havendo redução das dimensões previamente visualizadas. Por fim, pelo fato de não haver alterações em sistema renal e ausência de sinais, sintomas de insuficiência de adrenal, foi orientado o acompanhamento ultrassonográfico e observação de evolução clínica.

DISCUSSÃO

A correlação entre alterações de orelha externa e malformações congênitas renais é bem descrita e evidenciada na literatura atual através de estudos transversais, em diferentes populações, sendo a fisiopatologia ainda não explicada. ${ }^{9-11}$

A incidência de tais malformações aumenta quando associados fatores de risco para defeito na organogênese fetal $e$ embriogênese. Recém-nascidos de mães diabéticas possuem quatros vezes mais chances de apresentarem anomalias congênitas do que não diabéticas, havendo relação direta com nível de hemoglobina glicada. A exposição fetal à citocinas inflamatórias presentes em gestantes tabagistas e hipertensas impacta direta e indiretamente o feto. ${ }^{12}$

Mudanças embriológicas da fusão renal, sendo a mais frequente o rim em "ferradura", podem estar relacionadas a outras anormalidades urogenitais, as quais se apresentam assintomáticas inicialmente. Tais crianças são mais susceptíveis a complicações e desenvolvem sintomas decorrentes delas, como cálculos, infecção de repetição e obstrução urinária que resultam em hidronefrose, doenças renais crônicas e até quadros de sepses graves. ${ }^{13,14}$

Assim, a utilização da ultrassonografia, frente ao apêndice auricular isolado, deve ser levada em consideração quando associado a fatores maternos que aumentam a incidência de anomalias congênitas, principalmente tabagismo, hipertensão arterial e diabetes mellitus.

Além de ser um exame de imagem cada vez mais acessível aos serviços de saúde, não invasivo e com poucas limitações à sua realização, permite o diagnóstico precoce, sendo pedra angular do atual modelo de assistência à saúde. Se presente alterações orgânicas importantes, tais impactam de forma direta em todo o desenvolvimento, prognóstico e qualidade de vida do neonato, já que morbimortalidade relacionada a complicações renais é elevada.

REFERÊNCIAS

1. Krumar V, Abbas AK, Fausto N. Robbins e Cotran - Patologia: bases patológicas das doenças. 10. ed. Rio de Janeiro: Elsevier; 2018.

2. Fotter R. Pediatric uroradiology. 2. ed. Berlin: Springer-Verlag; 2008.

3. Fathallah-Shaykh SA, Flynn JT, Pierce CB, Abraham AJ, Blydt-Hansen TD, Massengill SF et al. Progression of pediatric CKD of nonglomerular origen in the CKiD cohort. Clin J Am Soc Nephrol 2015;10:571-77.

4. Wang RY, Earl DL, Ruder RO, Graham JM Jr. Syndromic ear anomalies and renal ultrasounds. Pediatrics. 2001;108(2):E32.

5. Kugelman A, Tubi A, Bader D, Chemo $M$, Dabbah $\mathrm{H}$. Pre-auricular tags and pits in the newborn: the role of renal ultrasonography. $\mathrm{J}$ Pediatr. 2002;141(3):388-91.

6. Hawdon JM. Babies born after diabetes in pregnancy: what are the short- and long-term risks and how can we minimise them? Best Pract Res Clin Obstet Gynaecol. 2011;25(1): 91-104. 
7. Villar J, Cheikh I, Victora C, Ohuma E, Bertino E, Altman DG et al. International Fetal and Newborn Growth Consortium for the 21st Century (INTERGROWTH-21st) International standards for newborn weight, length, and head circumference by gestational age and sex: the Newborn Cross-Sectional Study of the INTERGROWTH-21st Project. Lancet. 2014;384:857-68.

8. Kalhan SC, Parimi PS, Lindsay CA. Pregnancy complicated by diabetes mellitus. In: Fanaroff AA, Martin RJ (ed.) Neonatal-perinatal medicine: diseases of the fetus and infant. 7.ed. Philadelphia: Mosby; 2002.

9. Scheinfeld NS, Silverberg NB, Weinberg JM, Nozad V. The preauricular sinus: a review of its clinical presentation, treatment, and associations. Pediatr Dermatol. 2004;21(3): 191-96.

10. Roth DA, Hildesheimer M, Bardenstein S, Goidel D, Reichman B, Maayan-Metzger A et al. Preauricular skin tags and ear pits are associated with permanent hearing impairment in newborns. Pediatrics. 2008;122(4):e884-90.

11. Andrés-Jensen L, Jørgensen FS, Thorup J, Flachs J, Madsen JL, Maroun LL et al. The outcome of antenatal ultrasound diagnosed anomalies of the kidney and urinary tract in a large Danish birth cohort. Arch Dis Child. 2016;101(9):819-24.

12. Kicklighter SD. Infant of diabetic mothers. Pediatr Clin North Am. 2004;51:619-37.

13. Raj GV, Auge BK, Assimos D, Preminger GM. Metabolic abnormalities associated with renal calculi in patients with horseshoe kidneys. J Endourol. 2004;18(2):157-61.

14. Yavuz S, Kıyak A, Sander S. Renal outcome of children with horseshoe kidney: a single-center experience. Urology. 2015;85(2):463-66.

\section{CONFLITO DE INTERESSES}

Os autores declaram não haver conflitos de interesse

\section{AUTOR PARA CORRESPONDÊNCIA}

\section{Bruno Antunes Contrucci}

Pediatria, Hospital da Criança e Maternidade, Faculdade de Medicina de São José do Rio Preto (FAMERP)

15090-000 São José do Rio Preto - SP, Brasil

E-mail: bruno_acontrucci@hotmail.com

Submetido em 01/06/2021

Aceito em 16/07/2021 